\title{
Bufalin inhibits gastric cancer invasion and metastasis by down- regulating Wnt/ASCL2 expression
}

\author{
Jie Wang ${ }^{1, *}$, Han Cai ${ }^{1, *}$, Yue Xia ${ }^{1, *}$, Shiying Wang ${ }^{2}$, Likai Xing ${ }^{1}$, Chao Chen ${ }^{1}$, Yong \\ Zhang $^{1}$, Jie $X u^{1}$, Peihao Yin ${ }^{1}$, Yiming Jiang ${ }^{1}$, Ronghua Zhao ${ }^{3}$, Qingshong Zuo ${ }^{1}$ and \\ Teng Chen ${ }^{1,4}$ \\ ${ }^{1}$ Department of General Surgery, Putuo Hospital, Shanghai University of Traditional Chinese Medicine, Shanghai 200062, \\ China \\ ${ }^{2}$ Department of Gastroenterology, Longhua Hospital, Shanghai University of Traditional Chinese Medicine, Shanghai 200032, \\ China \\ ${ }^{3}$ Department of Medical, Virogin Biotech Ltd., Vancouver, British Columbia V6S 2L9, Canada \\ ${ }^{4}$ Shanghai Putuo Central School of Clinical Medicine, Anhui Medical University, Shanghai 200062, China \\ *These authors have contributed equally to this work \\ Correspondence to: Teng Chen, email: 13801842406@163.com \\ Qingshong Zuo, email: zqsads/123@sina.com \\ Keywords: bufalin; gastric cancer; invasion and metastasis; Wnt/ASCL2 signaling; EMT \\ Received: September 28, 2017 Accepted: January 02, 2018 Epub: January 11, 2018 Published: May 04, 2018 \\ Copyright: Wang et al. This is an open-access article distributed under the terms of the Creative Commons Attribution License 3.0 \\ (CC BY 3.0), which permits unrestricted use, distribution, and reproduction in any medium, provided the original author and source \\ are credited.
}

\section{ABSTRACT}

Achaete-scute-like 2 (ASCL2) is a transcription factor containing a basic helixloop-helix (bHLH) domain and is a downstream target of Wnt signaling in intestinal stem cells. Bufalin is the primary active ingredient in Chan Su, a traditional Chinese medicine obtained from the skin and parotid venom glands of toads. The purpose of this study was to research the anti-invasion and anti-metastasis activity of bufalin in gastric cancer and to identify the potential mechanism. Bufalin inhibited gastric cancer cell invasion and metastasis, suppressed cancer cell colony formation, and inhibited the growth of subcutaneous xenografted tumors in nude mice. Furthermore, bufalin inhibited ASCL2 expression and down-regulated the expression of invasionrelated genes such as MMP2, MMP9, and vimentin, thereby suppressing epithelialmesenchymal transition (EMT) in gastric cancer. A Wnt signaling inhibitor (XAV939) down-regulated invasion and the expression of ASCL2, $\beta$-catenin, and vimentin but up-regulated E-cadherin expression. In nude mice, bufalin inhibited the tumorigenic behavior of gastric cancer cells, induced cancer cell apoptosis, and regulated invasionrelated gene expression. Together, our results suggest that bufalin arrests invasion and metastasis and that its mechanism of action may involve down-regulating Wnt/ ASCL2 expression.

\section{INTRODUCTION}

Gastric cancer (GC) is one of the most common malignancies worldwide [1]. In China, GC ranks secondhighest in incidence and mortality [2]. Surgery is still the first choice for the treatment of GC. However, most patients are diagnosed at an advanced stage, and the available treatments are usually ineffective; more than half of radically resected GC patients experience local relapse or distant metastasis, and the majority receive a diagnosis of $\mathrm{GC}$ when the tumor has already disseminated. Thus, the median survival rarely exceeds 12 months, and the 5-year survival is less than $10 \%$ [3]. Therefore, it is of great significance to investigate the mechanism of GC progression and metastasis and to identify potent drugs or therapeutic targets.

Bufalin is a bioactive polyhydroxysteroid isolated from Venenum Bufonis, also called Chan Su, a traditional 
Chinese medicine obtained from the skin and parotid venom glands of toads [4]. Recent experimental studies have indicated that $\mathrm{Chan} \mathrm{Su}$ and its active compound bufalin have significant antitumor activity in various cancers. For example, bufalin can inhibit cancer cell proliferation $[5,6]$, induce cancer cell apoptosis and autophagy $[7,8]$, inhibit cancer metastasis and invasion $[9,10]$, and reverse multidrug resistance [11]. Studies had found that bufalin inhibits GC proliferation, induces apoptosis, and reverses cisplatin resistance [12, 13]. However, the anti-invasion and anti-metastasis activity of bufalin in GC is still not clear.

Achaete-scute-like 2 (ASCL2), a transcription factor containing a basic helix-loop-helix (bHLH) domain, is a member of the achaete-scute complex-like (ASCL) gene family, which is also referred to as the 'achaete-scute complex homolog' family, 'achaete-scute family basic helixloop-helix transcription factor', or mammalian achaetescute homologs (MASH); there are five family members (ASCL1-ASCL5) $[14,15]$. ASCL2 is a master regulator of intestinal stem cell identity and a Wnt target gene. ASCL2 also forms an autoactivating loop that results in an on/off expression pattern; this loop translates a Wnt gradient into a discrete transcriptional decision. In this manner, ASCL2 forms a transcriptional switch that is both Wnt responsive and Wnt dependent to define stem cell identity $[16,17]$. Studies have reported that abnormal ASCL2 expression is involved in cell proliferation, invasion, migration, and epithelial-mesenchymal transition (EMT) in colorectal cancer (CRC) [18, 19]. In addition, elevated ASCL2 expression is associated with the metastasis of osteosarcoma and lung squamous cell carcinoma and predicts poor prognosis in these patients [20, 21].

Data have also indicated that ASCL2 expression is up-regulated and markedly hypomethylated in GC tissues compared with adjacent normal tissues. ASCL2 expression levels were inversely correlated with GC patient survival. Ectopic overexpression of ASCL2 can increase cell growth and promote resistance to 5-fluorouracil in GC cells [22]. In our preliminary study, we screened a GC cell line with high ASCL2 expression and found that bufalin down-regulated ASCL2 expression. However, it remained unknown whether down-regulating ASCL2 expression affects GC invasion and metastasis and whether bufalin inhibits GC invasion and metastasis by regulating ASCL2 expression. Therefore, in this study, we down-regulated ASCL2 expression with lentiviral-based RNAi, observed the invasion and migration of transfected GC cells, and studied the effect of bufalin on GC both in vitro and in vivo.

\section{RESULTS}

\section{Bufalin inhibited gastric cancer proliferation and colony formation}

To study the effect of bufalin on GC, we used the most frequently used chemotherapeutic, oxaliplatin, as a positive control. MTT assay results showed that after treatment with different concentrations of bufalin $(0,50$, $100,200,400,1000$, or $2000 \mathrm{nM})$ or oxaliplatin $(0,1.25$, $2.5,5,10$, or $20 \mu \mathrm{M}$ ), the proliferation of GC cells (AGS) was obviously restrained, and cell growth was inhibited in a dose- and time-dependent manner. The half-inhibitory concentration (IC50) of bufalin at $48 \mathrm{~h}$ in AGS cells was approximately $72 \mathrm{nM}$, while that for oxaliplatin was 8.56 $\mu \mathrm{M}$ (Figure 1A). Therefore, we used these concentrations in our subsequent study. The colony formation experiment showed that bufalin and oxaliplatin inhibited GC colony clone, but there was no difference between bufalin and oxaliplatin (Figure 1B).

\section{Bufalin suppressed the invasion and metastasis ability of GC cells}

We used wound healing assays to analyze the impact of bufalin on GC cell migration. Migration was obviously suppressed after treatment with bufalin or oxaliplatin in $24 \mathrm{~h}, 48 \mathrm{~h}$ and $72 \mathrm{~h}$ compared with control group. But there was no difference between the bufalin and oxaliplatin group in $24 \mathrm{~h}, 48 \mathrm{~h}$, while in the time of $72 \mathrm{~h}$ the Oxaliplatin surpress function was more obviously than bufalin (Figure 1C). To further ascertain the effect of bufalin on tumor cell invasion and metastasis, we performed transwell experiments and found that cell invasion decreased after treatment with bufalin or oxaliplatin; however, oxaliplatin had a stronger effect (Figure 1D).

\section{ASCL2 promoted gastric cancer cell invasion and metastasis}

To study the function of ASCL2 in GC invasion and metastasis, a lentiviral vector containing RNAi targeting the coding region of human ASCL2 mRNA (ASCL2shRNA) was constructed. The vector was transfected into AGS cells, and ASCL2 expression was detected by western blotting. ASCL2 expression was obviously reduced in shRNA-ASCL2-transfected AGS cells (Figure 2A), and AGS cells with ASCL2 down-regulated were successfully constructed. The migration and invasion of the transfected AGS cells were evaluated using wound healing assays and transwell assays, respectively. Compared with the control vector, the ASCL2-shRNA vector obviously decreased the migration (Figure $2 \mathrm{~B}$ ) and invasion (Figure 2C) of AGS cells. The colony formation assay showed that down-regulation of ASCL2 expression markedly restrained AGS colony formation (Figure 2D).

\section{Bufalin down-regulated ASCL2 expression}

The above studies indicated that bufalin inhibits $\mathrm{GC}$ invasion and migration and that down-regulating ASCL2 expression has the same consequences. Therefore, the question arose as to whether there is any relation between bufalin-mediated anti-cancer activity and ASCL2 

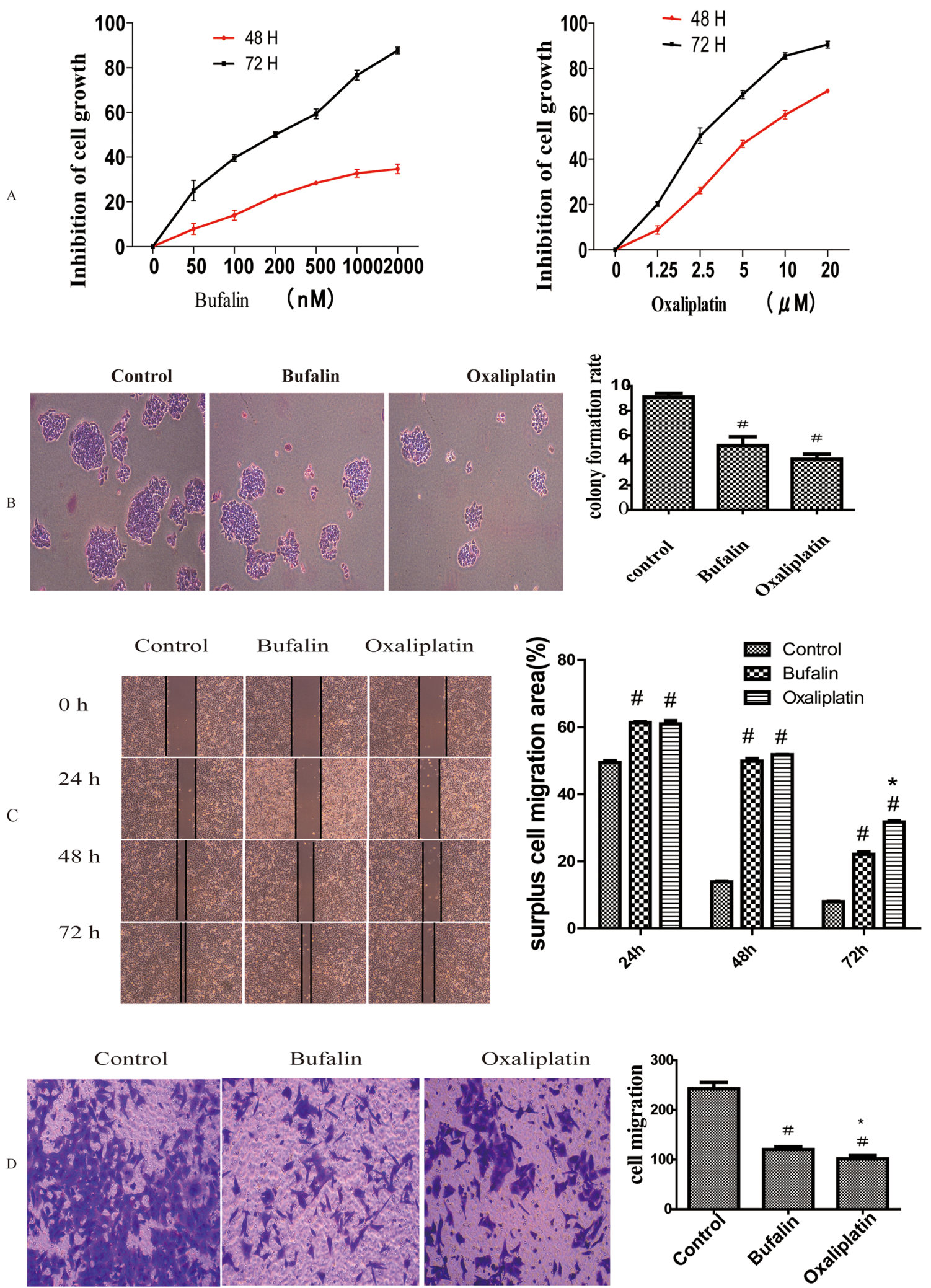

Figure 1: Effect of bufalin on GC cell proliferation and invasion (A) MTT assays showing the inhibitory effect of bufalin and oxaliplatin on cell proliferation. (B) Colony formation assays after treatment with bufalin, oxaliplatin, or normal saline (control). (C) Effect of bufalin and oxaliplatin on cell invasion in wound healing assays. (D) Effect of bufalin on cell invasion in transwell assays. All data are representative of at least three independent experiments. ${ }^{\sharp} P<0.01$ compared with the control group, ${ }^{*} P<0.05$ compared with the bufalin group. 

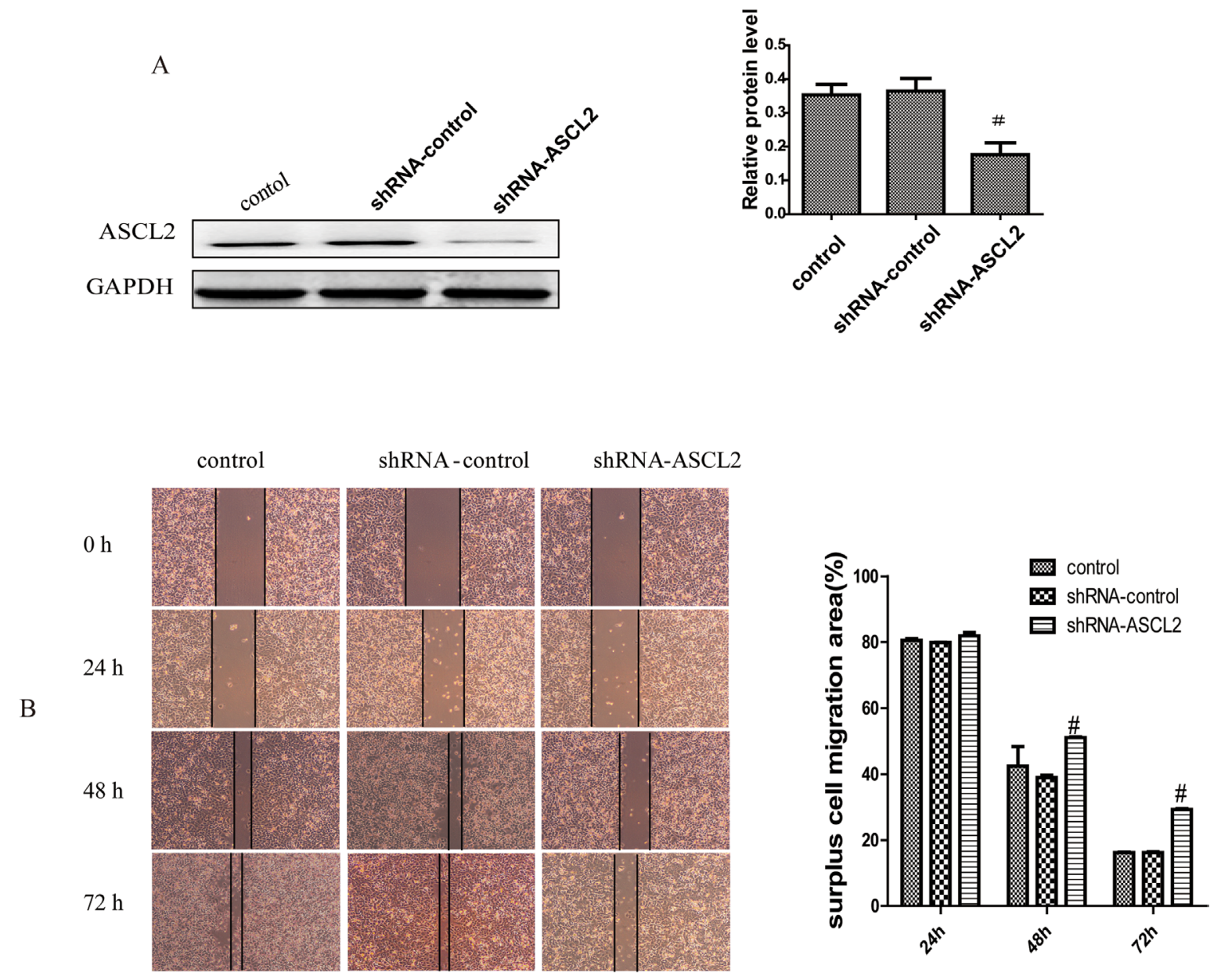

control

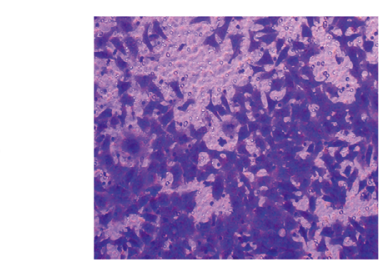

shRNA - control

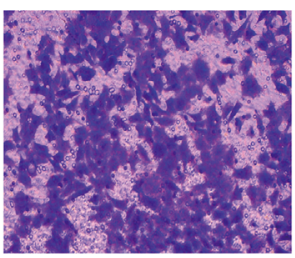

$\mathrm{C}$

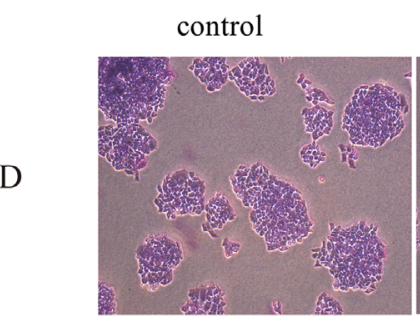

shRNA-control

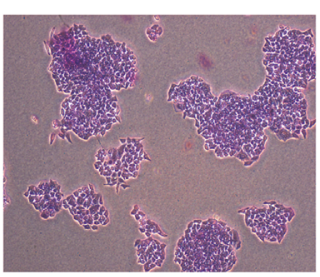

ShRNA - ASCL2
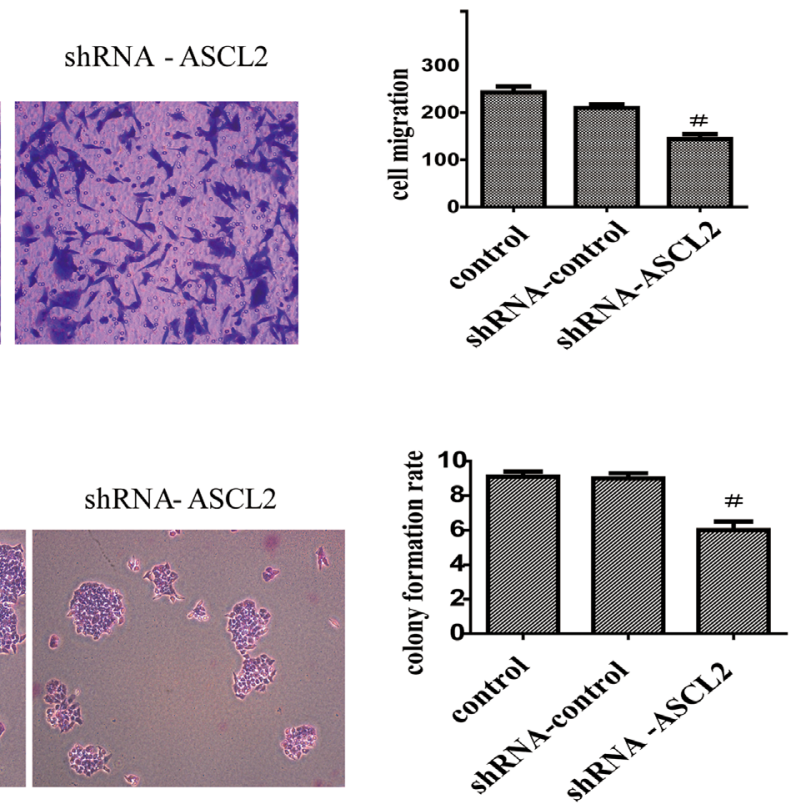

Figure 2: ASCL2 promotes gastric cancer cell invasion and metastasis (A) ASCL2 expression after transfection of ASCL2-shRNA. (B-C) Effect of down-regulating ASCL2 on cell invasion in wound healing and transwell assays. (D) Effect of ASCL2 down-regulation on colony formation ability. All data are representative of at least three independent experiments. ${ }^{*} P<0.05$ compared with the control group. 
expression. Thus, we determined the effect of bufalin on ASCL2. Western blotting and RT-PCR results showed that bufalin treatment down-regulated ASCL2 expression, and this effect was enhanced by co-treatment with shRNAASCL2. Furthermore, bufalin and oxaliplatin had the same activity; co-treatment with both compounds further decreased ASCL2 expression (Figure 3B).

\section{Bufalin inhibited GC cell invasion and metastasis by suppressing EMT}

Since RNAi targeting ASCL2 inhibited the invasion and migration of GC cells, we studied the underlying mechanism. Western blot results showed that ASCL2 down-regulation inhibited the expression of MMP2 and MMP9, increased the expression of the epithelial marker E-cadherin and decreased the expression of the mesenchymal marker vimentin (Figure 3C). Western blot assays were performed to determine whether bufalin affects these genes and showed that bufalin treatment had

A
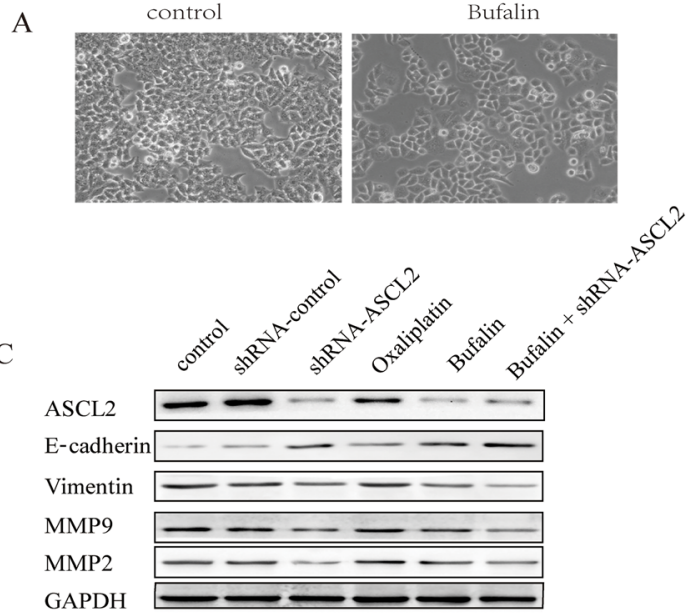

Bufalin

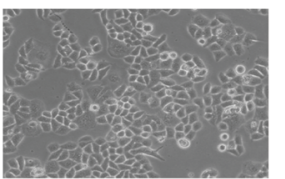

control

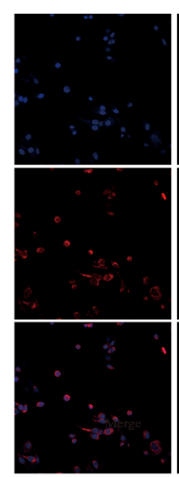

shRNA-ASCL2

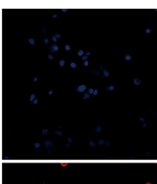

Bufalin

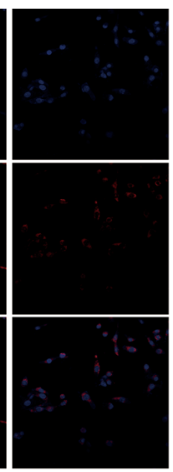

Bufalin+shRAN-ASCL2

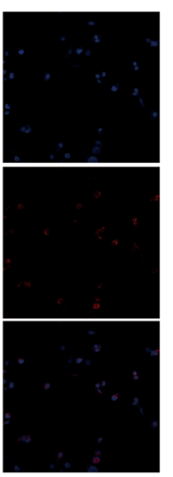

the same outcome as RNAi targeting ASCL2; combined treatment of bufalin and shRNA-ASCL2 led to a further increased in E-cadherin expression and a further decrease in MMP2, MMP9 and vimentin expression (Figure 3C).

Immunofluorescent detection demonstrated decreasing expression of vimentin and an increasing expression of E-cadherin in the shRNA-ASCL2 AGS cells and bufalin treated cells compared with the control group. Combination treatments with bufalin and shRNAASCL2 had greater effects than the single agents(Figure 3D). Moreover, morphological changes were evident after bufalin treatment; GC cells lost their fibroblastlike appearance and acquired an epithelial morphology (Figure 3A).

\section{Bufalin inhibited the Wnt/ $\beta$-catenin signaling pathway}

Studies have shown that ASCL2 is a master regulator of intestinal stem cell identity and a Wnt target gene [16].
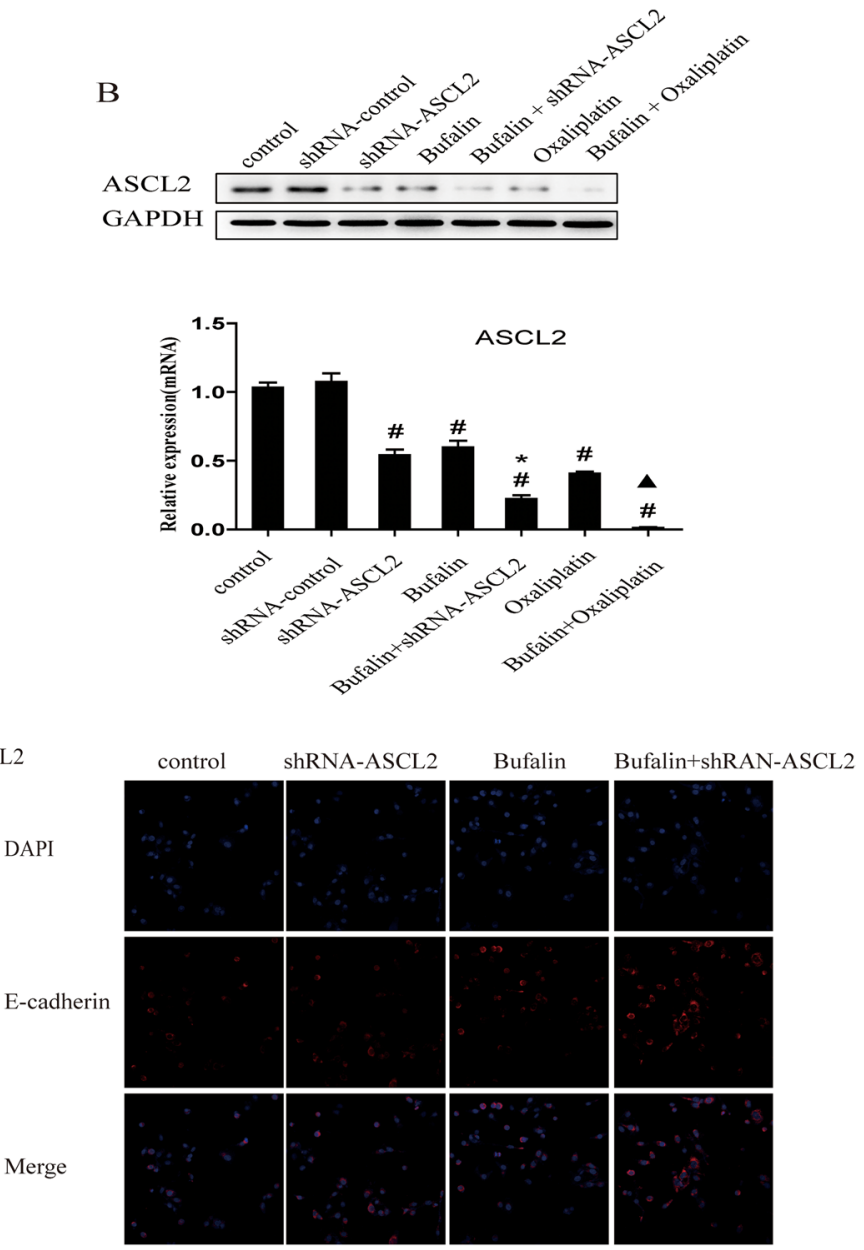

Figure 3: Effect of bufalin on ASCL2 and EMT. (A) Morphological changes after bufalin treatment. (B) Bufalin down-regulated ASCL2 expression both in protein and mRNA. (C) Bufalin affected invasion and metastasis by suppressing EMT. (D) Immunofluorescent detection of vimentin and E-cadherin expression, Nucleus were counterstained using DAPI. 
Therefore, we inhibited Wnt signaling with XAV939 to determine whether this influences ASCL2 expression. Western blot results showed that after XAV939 treatment, $\beta$-catenin and ASCL2 expression were down-regulated, while the expression of the EMT-related gene E-cadherin was up-regulated (Figure 4A). Transwell assays showed that GC cell invasion was inhibited when Wnt signaling was blocked (Figure 4B). Next, we treated GC cells with bufalin and observed that ASCL2, $\beta$-catenin and vimentin expression was down-regulated; the down-regulation of these genes was more obvious when bufalin was combined with XAV939 (Figure 4A), and E-cadherin expression was higher in cells treated with the combination than in those treated with bufalin only (Figure 4A). Transwell assays also showed stronger inhibition of cell invasion by bufalin combined with the Wnt inhibitor than by bufalin alone (Figure 4B).

\section{The effect of ASCL2 and Bufalin on tumorigenic ability}

To further investigate ASCL2 function and bufalin activity in GC, we generated a subcutaneous transplant model in nude mice (Figure 5A). First, we observed the effect of ASCL2 on the tumorigenic ability of GC cells; tumorigenesis was markedly inhibited upon downregulation of ASCL2 expression (Figure 5B). The tumor volume was smaller and the tumor weight was lighter compared with the values in the control group (Figure 5C-5D). Then, we injected bufalin intraperitoneally into animals in the normal cancer cell group and the shRNAASCL2 cell group; the control group received normal saline (NS), and the positive control group received oxaliplatin. Bufalin and oxaliplatin inhibited the growth of transplanted tumors compared with control and shRNAASCL2, but there was no obvious difference between the bufalin and oxaliplatin groups (Figure 5A-5D). In addition, compared with the groups that received bufalin or oxaliplatin alone, the shRNA-ASCL2 combination treatment groups had smaller and lighter tumors (Figure $5 \mathrm{~A}-5 \mathrm{D})$.

\section{The effect of bufalin on invasion- and metastasis- related genes in vivo}

Next, we observed the effect of bufalin on ASCL2 and related genes in mouse tumors. Western blot analysis showed that ASCL2, MMP2 and vimentin expression
A

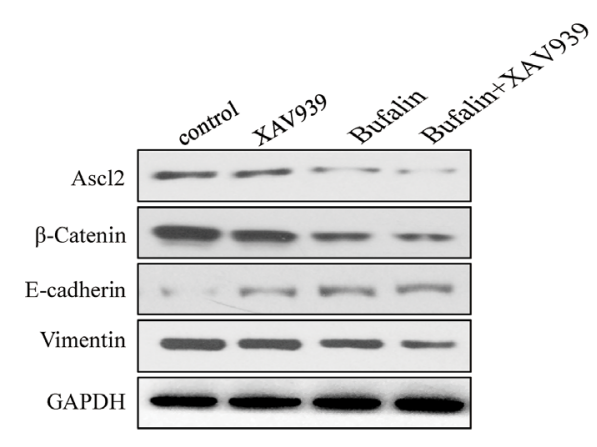

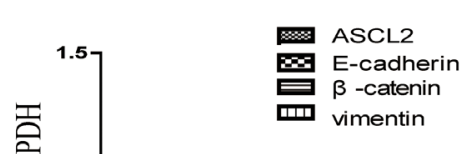
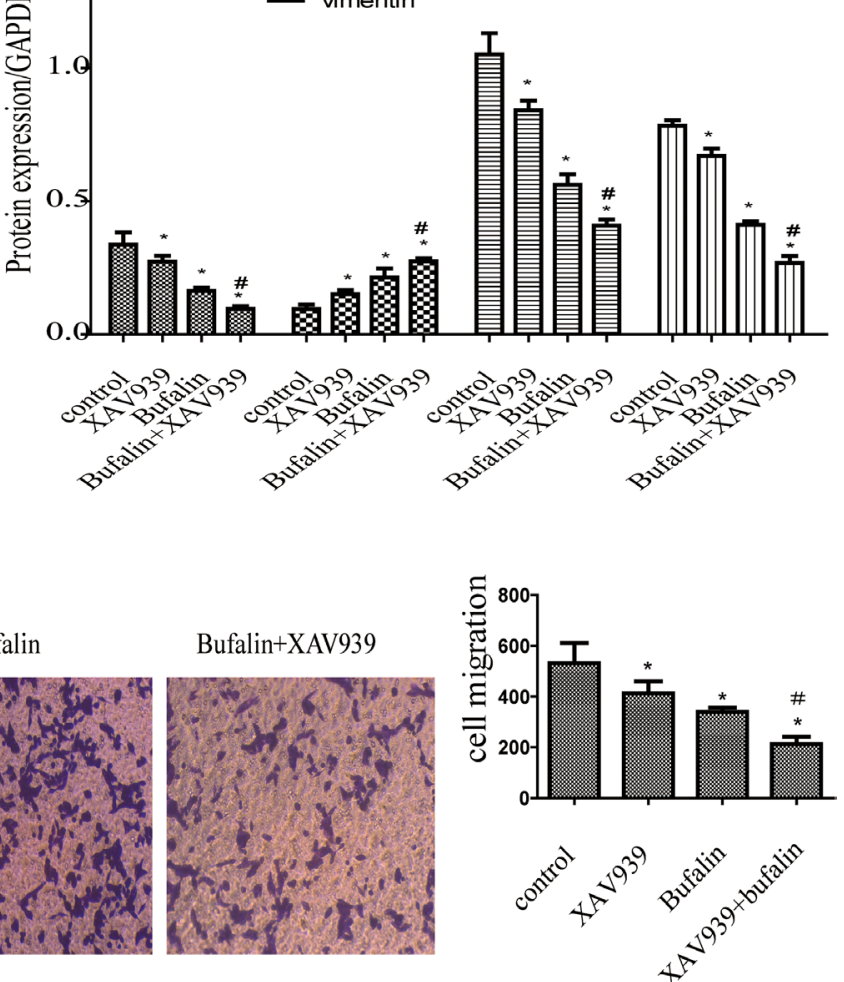

Figure 4: Bufalin inhibits the Wnt/ $\beta$-catenin signaling pathway. (A) The effect of bufalin and XAV939 on ASCL2, $\beta$-catenin, E-cadherin and vimentin expression. (B) The effect of bufalin and XAV939 on gastric cancer invasion. All data are representative of at least three independent experiments. ${ }^{*} P<0.01$ compared with the control group, ${ }^{\#} P<0.05$ compared with the XAV939 and Bufalin groups. 

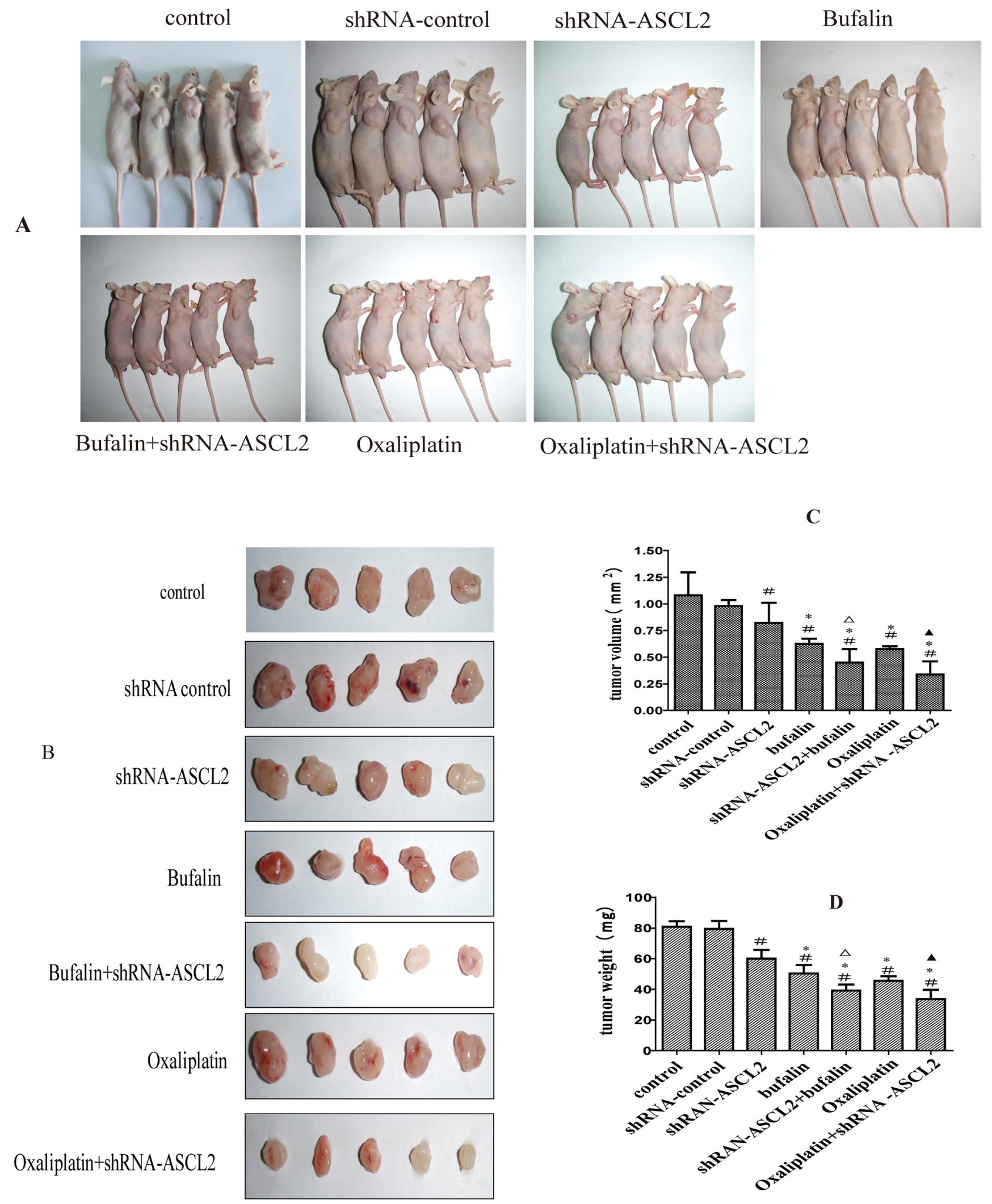

Figure 5: The effect of bufalin on tumorigenic activity (A) Subcutaneous transplant model in nude mice. (B) Tumors extracted after subcutaneous growth. (C) Tumor volume of each group. (D) Tumor weight of each group. ${ }^{*} \mathrm{P}<0.01$ compared with the control group, ${ }^{*} \mathrm{P}<0.05$ compared with the shRNA-ASCL2 group, ${ }^{\triangle} P<0.05$ compared with the bufalin group, ${ }^{\wedge} P<0.01$ compared with the oxaliplatin group. 
were down-regulated after treatment with bufalin and oxaliplatin (Figure 6A), and these results were verified by immunohistochemistry (IHC) (Figure 6B). Moreover, when bufalin or oxaliplatin was combined with shRNAASCL2, MMP2 and vimentin expression was further inhibited, and MMP9 expression was down-regulated; however, there were no difference between bufalin and oxaliplatin (Figure 6A). We also examined E-cadherin expression in the tumors; western blots showed that E-cadherin was up-regulated in the bufalin, oxaliplatin group and shRNA-ASCL2 groups (Figure 6A). Combination treatments with shRNA-ASCL2 had greater effects than the single agents (Figure 6A).

\section{Bufalin induced gastric cancer apoptosis}

Finally, we ascertained the effect of bufalin on GC apoptosis. TUNEL assay results showed that downregulating ASCL2 expression induced GC apoptosis; the apoptosis rate was higher in the shRNA-ASCL2 group than in the control group. In addition, the apoptosis rate in the bufalin and oxaliplatin groups was higher than that in the control group, while combination treatments with shRNA-ASCL2 led to increased apoptosis compared with bufalin or oxaliplatin alone (Figure 6C). Then we examed the expression of apoptosis relate genes, western-blot result showed that down-regulating ASCL2 expression or treat with bufalin can inhibite the expression of Bcl2, and up-regulate the expression of Bax and Caspase- 3 . When combination treatments with shRNA-ASCL2 and bufalin the expression of Bcl-2 almost died out, while the expression of Bax and Caspase- 3 were much higher (Figure 6D).

\section{DISCUSSION}

Bufalin is a bioactive polyhydroxysteroid isolated from Venenum Bufonis, also called Chan $\mathrm{Su}$, a wellknown traditional Chinese medicine widely used in cancer treatment in China [4, 23]. Recent studies have shown that bufalin has anti-cancer activity in various cancers, such as breast cancer [24], osteosarcoma [5], colon cancer [11, 25], lung cancer [26], pancreatic cancer [27], bladder cancer [28] and hepatocellular carcinoma [9]. Studies had found that bufalin inhibits GC proliferation, induces apoptosis and reverses cisplatin resistance [12, 13]. However, the effects of bufalin on GC invasion and metastasis were not clear. In this study, we found that bufalin inhibits GC proliferation and metastasis. Bufalin treatment decreased colony formation, invasion and migration (Figure 1). In the vivo study, bufalin inhibited the growth of subcutaneous transplanted tumor cells in a nude mouse model (Figure 5). In this study, we used oxaliplatin as a positive control. We observed that bufalin had the same effects as oxaliplatin on GC, but the dose of bufalin was much lower; therefore, bufalin may have potential as a chemotherapeutic inhibitor of GC invasion and metastasis.

Ascl2 is a master regulator of intestinal stem cell identity and a Wnt target gene [16, 17]. Studies have shown that abnormal expression of ASCL2 can enhance the invasion and metastasis of CRC cells in vitro; that overexpression of ASCL2 correlates with distant metastasis, tumor size and poor overall survival in CRC patients; and that high expression of ASCL2 promotes EMT in CRC [18, 19, 29]. In addition, elevated ASCL2 expression is associated with the metastasis of osteosarcoma and lung squamous cell carcinoma and predicts poor prognosis of these patients [20, 21]. A previous study found that ASCL2 expression was upregulated and markedly hypomethylated in GC tissues and that ectopic overexpression of ASCL2 increased cell growth and promoted resistance to 5-fluorouracil in GC cells [22]. In our study, we first determined the effect of ASCL2 on GC invasion and metastasis. Down-regulating ASCL2 expression with lentiviral vector-based RNAi inhibited GC cell colony formation. Wound healing and transwell assays showed that ASCL2 down-regulation led to decreased GC cell invasion and migration (Figure 2). Furthermore, down-regulating ASCL2 decreased the tumorigenicity of GC cells, and tumor volume and weight were both reduced (Figure 5).

Therefore, to investigate whether bufalin-mediated inhibition of GC cell invasion and metastasis is related to ASCL2 expression, we detect ASCL2 protein after treatment with bufalin. ASCL2 was obviously downregulated after cells were treated with bufalin and oxaliplatin. In addition, when these compounds were combined with shRNA-ASCL2, the expression of ASCL2 was nearly abolished (Figure 3B). Therefore, we speculate that bufalin inhibits GC cell invasion in a manner related to down-regulating ASCL2 expression.

Invasion and metastasis are the primary reasons for the poor prognosis of GC patients [3]. Similar to other cancers, GC cells degrade the basement membrane and extracellular matrix and migrate into adjacent areas before intravasating into blood and/or lymphatic vessels, circulating to the target organ site, extravasating into target organ tissue and proliferating in target organs. These steps are mediated by various factors and processes, including growth factors, proteolytic degradation of extracellular matrix, cell-cell adhesion, cytoskeleton remodeling and changes in gene expression [30]. MMP2 and MMP9 are members of the matrix metalloproteinase (MMP) family of proteolytic enzymes, and a previous study reported that MMPs are considerably elevated in malignant tumors [31]. Expression of these enzymes is a major characteristic of the malignant invasion and metastasis of cancer cells, as these enzymes degrade the ECM and may promote the penetration of cancer cells into the basement membrane [32]. Our study showed that bufalin and ASCL2 knockdown both inhibited the expression of MMP2 and 

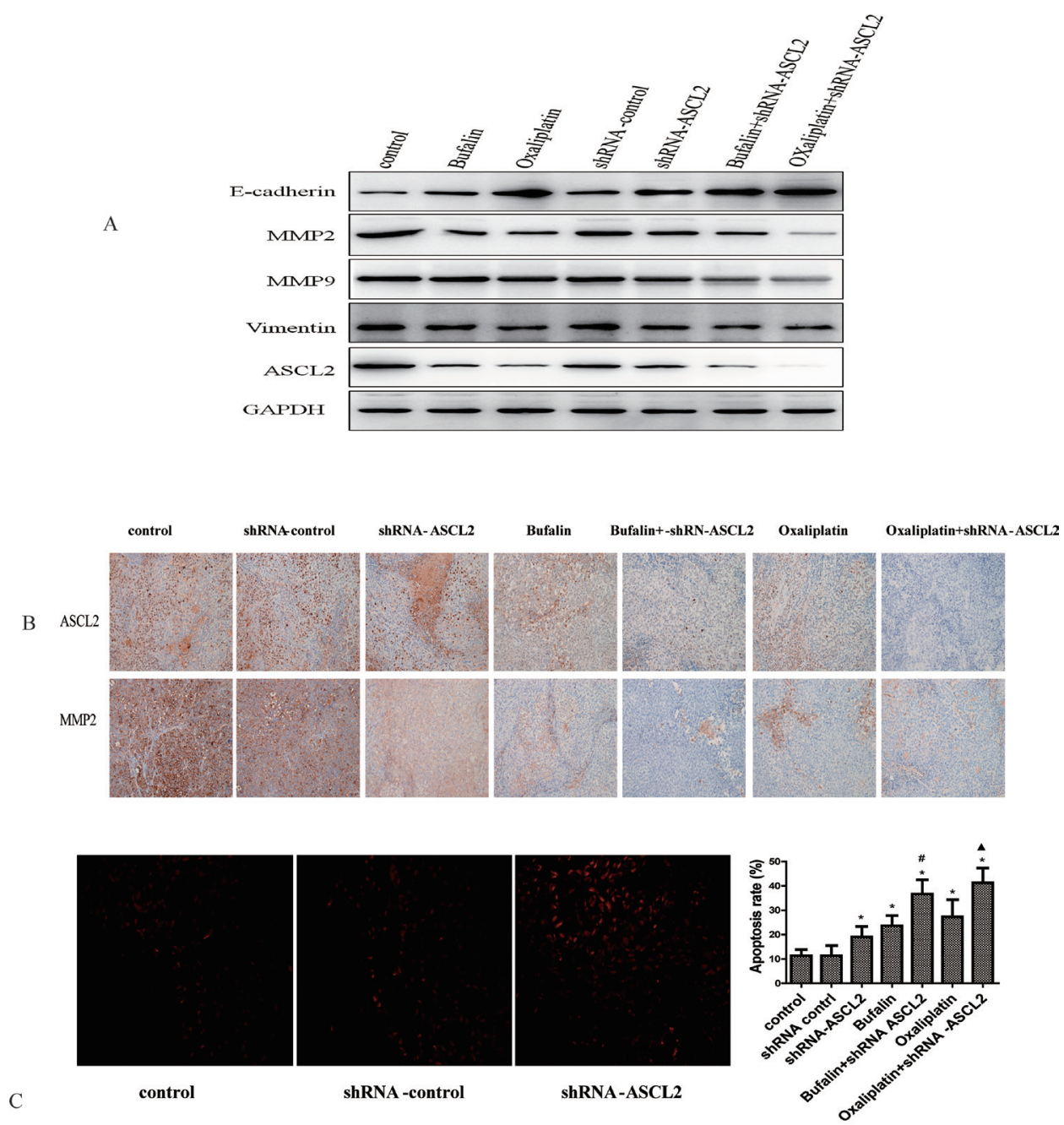

C

control

shRNA-control

shRNA-ASCL2
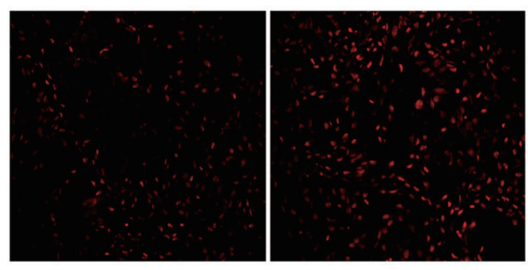

Bufalin+shRN-ASCL2

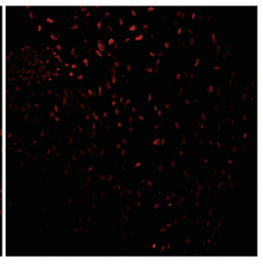

Oxaliplatin

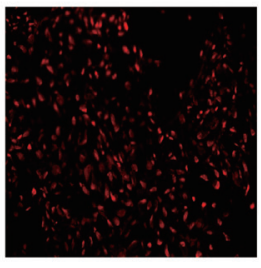

Bufalin
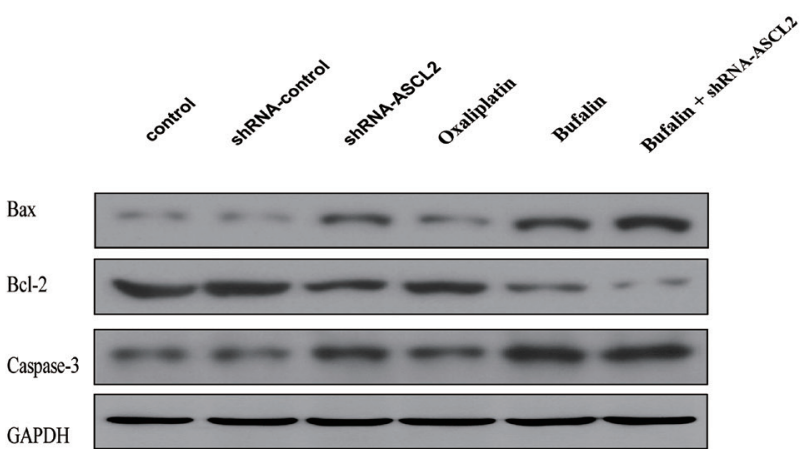

Figure 6: The effect of bufalin on related genes and apoptosis in vivo (A) Invasion- and metastasis-related genes expression in vivo (western-blot). (B) ASCL2 and MMP2 exprssion in tumor tissue (IHC). (C) The effect of bufalin on GC apoptosis (TUNEL). (D) The effect of bufalin on apoptosis relate genes of Bcl-2, Bax and Caspase-3. ${ }^{*} P<0.05$ compared with the control group, ${ }^{\#} \mathrm{P}<0.01$ compared with the bufalin group, $\triangle P<0.01$ compared with the oxaliplatin group. 
MMP2, with more pronounced results upon combining bufalin and shRNA-ASCL2 (Figure 3C); these results were verified in animal experiments (Figure 6A).

Another reason for metastasis is EMT, one of the key processes in cancer progression and metastasis. Upon undergoing EMT, cancer cells lose cell-cell connections, cell-matrix contact, and normal epithelial polarity while gaining mesenchymal characteristics to enable migration and invasion into the surrounding matrix [33-35]. During EMT, the expression of epithelial cell markers such as E-cadherin is down-regulated, while mesenchymal markers such as vimentin and $\mathrm{N}$-cadherin are up-regulated. Vimentin, a member of the type III intermediate filament (IF) protein family, plays an important role in maintaining cell shape and integrity and is involved in cytoskeletal interactions such as adhesion and migration [36].

In this study we found GC cells lost their fibroblastlike appearance and acquired an epithelial morphology after treated with bufalin. Therefore, we also evaluated the expression of the EMT-relate genes E-cadherin and vimentin. Western blot analysis showed that bufalin increased E-cadherin expression and down-regulated vimentin expression; these results were more pronounced when cells were co-treated with bufalin and shRNAASCL2 (Figure 3C). Immunofluorescent detection also showed shRNA-ASCL2 and bufalin can increased E-cadherin expression and down-regulated vimentin expression (Figure 3D). Therefore, we speculated that bufalin suppresses EMT by down-regulating ASCL2 expression.

Wnt/ $\beta$-catenin signaling pathways are crucial for cellular maintenance and development, include cell cycle progression, apoptosis, differentiation, migration and proliferation [37]. In addition, stimulation of these pathways is closely related to cancer development. During neoplastic transformation, the Wnt/ $\beta$-catenin pathway is often aberrantly activated, increasing migration and invasion potential through increased $\beta$-catenin phosphorylation and/or nuclear localization [38]. Research suggested that Ascl2 is a master regulator of intestinal stem cell identity - deletion of the Ascl2 gene in the adult small intestine led to the disappearance of Lgr5 stem cells within day — and a Wnt target gene [16, 17, 39]. Therefore, we inhibited the Wnt signaling pathway with XAV939, which down-regulated $\beta$-catenin and ASCL2 and upregulated E-cadherin; combining XAV939 with bufalin further decreased the expression of $\beta$-catenin and ASCL2 (Figure 4A). We also found that the migration ability of GC cells obviously decreased after XAV939 treatment, which increased the anti-invasion activity of bufalin (Figure 4B).

To explore the mechanism underlying the anti-GC activity of bufalin, we evaluated the effect of bufalin and ASCL2 on GC apoptosis in vivo and in vitro. In vivo study we found Bufalin decreased ASCL2 expression in the transplanted tumors and induced gastric cancer xenograft apoptosis. Then we detected the expression of apoptosis relate genes Bax, Bcl-2 and Caspase-3. We found after treated with Bufalin or down-regulate ASCL2 expression can increase Bax and Caspase-3 expression, at the same time down-regulated the expression of Bcl-2. And with more pronounced results upon combining bufalin and shRNA-ASCL2. Therefore, we inferred that bufalin inhibits GC tumorigenic behavior in vivo by influencing both cell proliferation and apoptosis, but the mechanism requires additional research.

Together, this study revealed that bufalin has antiinvasion and anti-metastasis activity in GC, and the underlying mechanism may relate to down-regulation of the Wnt/ $\beta$-catenin signaling pathway, followed by the inhibition of ASCL2 expression and EMT. However, this mechanism requires additional elucidation and confirmation in future studies.

\section{MATERIALS AND METHODS}

\section{Cell culture and materials}

The human GC cell line AGS was obtained from the Shanghai Institutes for Biological Sciences and cultured in RPMI-1640 medium supplemented with $10 \%$ FBS (Gibco USA), 100 units $/ \mathrm{ml}$ penicillin, $100 \mu \mathrm{g} / \mathrm{ml}$ streptomycin, and $2 \mathrm{mM}$ glutamine at $37^{\circ} \mathrm{C}$ with $5 \% \mathrm{CO}_{2}$. Bufalin (Sigma, USA) was dissolved in absolute ethyl alcohol and diluted in PBS. Oxaliplatin was obtained from Sanofi-Aventis (France); antibodies against E-cadherin, vimentin, MMP2, MMP9, and GAPDH were purchased from Cell Signaling Technology (Massachusetts, USA); and the ASCL2 antibody was obtained from Millipore (Massachusetts, USA).

\section{MTT assays}

Cell proliferation was measured using the Cell Proliferation Reagent Kit I (MTT) (Roche, Basel, Switzerland). Logarithmic phase GC cells were seeded into 96 -well plates $\left(1 \times 10^{4} /\right.$ well $)$ in maintenance medium and then treated with different concentrations of bufalin $(0,50,100,200,400,1000$, or $2000 \mathrm{nM})$ or oxaliplatin $(0,1.25,2.5,5,10$, or $20 \mu \mathrm{M})$; each concentration was evaluated in six wells. Cell proliferation was monitored at $0,24,48$ and $72 \mathrm{~h}$ according to the manufacturer's instructions.

\section{Colony formation assays}

The colony formation assay was performed as follows. Briefly, logarithmic phase GC cells were seeded into $6 \mathrm{~cm}$ dishes at approximately 2000 cells per dish, cultured at $37^{\circ} \mathrm{C}$ with $5 \% \mathrm{CO}_{2}$ for 14 days, fixed in $4 \%$ formalin for $15 \mathrm{~min}$, stained with Giemsa stain for $30 \mathrm{~min}$, washed in water and dried. The colonies were observed 
by microscopy and qualified with ImageJ software. The colony formation rate was calculated using the following formula: colony formation rate $(\%)=$ colonies/plated cells $\times 100 \%$.

\section{Wound healing assays}

Wound healing assays were conducted as previously reported [40]. A wound was created in a confluent layer of cells using a $200-\mu \mathrm{l}$ pipette tip, and floating cells were removed by washing with $\mathrm{PBS}$. The medium was replaced with fresh medium. Cells were subjected to the indicated treatment for 24,48 or $72 \mathrm{~h}$, and cells that migrated from the leading edge were photographed. Surplus cell migration area were analysed.

\section{Transwell assays}

Cell migration and invasion were observed in transwell assays. Briefly, after treating cells with related drugs or interventions for $48 \mathrm{~h}$, transwell assays were performed. First, the cells were adjusted to approximately $1 \times 10^{5}$ cells $/ \mathrm{ml}$ in serum-free medium, $150 \mu \mathrm{l}$ of the cell suspension was added to the upper chamber, and $600 \mu \mathrm{l}$ of DMEM containing 20\% FBS was added to the lower chamber. The cells were incubated in the transwells for $48 \mathrm{~h}$, and the transwell insert was then removed. A cotton-tipped applicator was used to carefully remove the cells that had not migrated/invaded from the top of the membrane. The membrane was washed twice with PBS, fixed with $3.7 \%$ formaldehyde for $15 \mathrm{~min}$, and stained with crystal violet. The cells were observed and photographed under a light microscope.

\section{Stable shRNA-mediated knockdown of ASCL2 in cell lines}

To knock down ASCL2, an shRNA sequence (5'-GCGTGAAGCTGGTGAACTTGG-3') were designed and synthesized by GenePharma (Shanghai, China). A scramble shRNA was used as a negative control. The sequences were cloned into the lentiviral pLKO.1 puro vector, which was purchased from GenePharma (Shanghai, China). Lentiviral production and transduction were performed according to the manufacturer's protocol. Then, stable cells expressing lower ASCL2 levels were cultured, and western blot assays were used to verify the knockdown.

\section{Western blot}

Total protein was extracted in RIPA buffer supplemented with protease and phosphatase inhibitors (Beyotime, JiangShu, China). Protein concentration was determined using the BCA protein concentration determination kit (Beyotime, JiangShu, China). Protein from the control and treated cell lysates was loaded onto
8-12\% gradient NuPAGE gels (Novex, San Diego, CA, USA), electrophoresed under reducing conditions, and transferred onto polyvinylidene difluoride membranes (0.22 $\mu \mathrm{m}$; Millipore, Billerica, USA). Western blot analysis was performed as described previously [25]. ASCL2 Monoclonal Antibody (MAB4417, Millipore, Billerica, USA), E-cadherin, vimentin, MMP2, MMP9, Bcl-2, Bax, Caspase-3 and GAPDH antibody (Cell Signaling Technology, MA, USA) were used.

\section{RNA isolation and quantitative RT-PCR}

Total RNA was extracted using the TRIzol Reagent (Invitrogen, Carlsbad, CA, USA), and total RNA was reverse-transcribed with the PrimeScript RT-PCR Kit (TakaraBio, Otsu, Japan). The RT-PCR was performed using $\mathrm{SYBR}^{\circledR}$ Premix Ex Taq $^{\mathrm{TM}}$ II (Takara Bio, Otsu, Japan). The ABI 7500 real-time PCR system (Applied Biosystems, Irvine, CA, USA). The primer sequences used were as follows: forward 5'GCGTGAAGCTGGTGAACTTG-3' and reverse 5'-GGATGTACTCCACGGCTGAG-3' for ASCL2; forward 5'-GGAGCGAGATCCCTCCAAAAT' and reverse 5'-GGCTGTTGTCATACTTCTCATGG-3' for GAPDH. The $2^{-\triangle \Delta C T}$ equation was used to calculate the relative expression levels.

\section{Immunofluorescence}

Briefly, cells were fixed with 4\% paraformaldehyde and permeabilized with $0.1 \%$ Triton X-100 in PBS. Following cell fixation, cells were incubated with the appropriate primary antibodies (vimentin and E-cadherin) in a solution of PBS with $1 \%$ bovine serum albumin and $0.1 \%$ Triton $\mathrm{X}-100$ at $4{ }^{\circ} \mathrm{C}$ overnight. Cells were further incubated with Alexa Fluor 488 labeled secondary antibody for 1 hour at room temperature, nuclei were stained with DAPI for $5 \mathrm{~min}$. Fluorescent signals were detected by confocal fluorescence microscopy (LEICA DM IRB; Leica, Wetzlar, Germany)

\section{In vivo study}

For the in vivo studies, 5- to 6-week-old male $\mathrm{BALB} / \mathrm{c}$ mice (18-20 grams) were purchased from the Shanghai Laboratory Animal Center (SLACCAS, Shanghai, China). The mice were housed in a temperatureand humidity-controlled, pathogen-free animal facility and fed according to standard instructions provided by SLACCAS. All animal studies were performed in accordance with the International Standards of Animal Welfare and were approved by the Institute of Animal Care and Use Committee of Shanghai University of Traditional Chinese Medicine (approval number: SYXK, Shanghai, 2013-0055).

Gastric AGS cells, shRNA-control AGS cells, and shRNA-ASCL2 AGS cells were harvested from 
culture flasks and suspended in culture medium at a concentration of $1 \times 10^{7}$ cells $/ \mathrm{ml}$. For each mouse, $0.2 \mathrm{ml}$ of the cell suspension was injected subcutaneously into the right axillary region. After 3 weeks, the mice were randomly divided into the control group (AGS), shRNAcontrol group, shRNA-ASCL2 group, bufalin group, bufalin+shRNA-ASCL2 group, oxaliplatin group and oxaliplatin+shRNA-ASCL2 group, with at least five mice in each group. The intervention groups were treated with bufalin or oxaliplatin via intraperitoneal injection twice per week for 3 weeks. The mice were then sacrificed, and the tumors were excised. The tumor weight, maximum diameter $(a)$ and minimum diameter $(b)$ were measured, and the tumor volume $(V)$ were calculated as follows: volume $=a^{2} b / 2$. Tumor tissue was stored in liquid nitrogen .

\section{Immunohistochemistry (IHC)}

Immunohistochemical analysis was performed as described previously [25]. Briefly, tumor sections were stained with mouse anti-ASCL2 or mouse anti-MMP2 antibody at $4^{\circ} \mathrm{C}$ overnight. Goat anti-rabbit or antimouse $\mathrm{IgG} /$ horseradish peroxidase was applied as the secondary antibody according to the standard protocols provided by the manufacturer of the Elivision ${ }^{\mathrm{TM}}$ plus kit (Maixin Bio, Fuzhou, China). For negative controls, the primary antibody was replaced with PBS. Five high power microscopic fields $(\times 200)$ were randomly chosen from each slide to determine the positive staining intensity by IPP software (Image-Pro Plus 6.0, Media, Cybernetics). An unstained region was selected and set as the background. The protein expression level is presented as the average staining intensity of the 5 fields from each slide.

\section{Apoptosis assays}

To determine apoptosis in tissue samples, TUNEL assays (terminal deoxynucleotidyl transferase deoxyUTP-fluorescein nick end labeling assay) were performed. Tissues sections ( $4 \mu \mathrm{m})$ were evaluated using the In Situ Cell Death Detection Kit (Roche, Mannheim, Germany) according to the procedures described by the manufacturer. Under a fluorescence microscope, apoptotic cells emit red fluorescence; 5 randomly chosen fields $(\times 200)$ without any necrotic areas were observed, and the average percentage of positive cells in the 5 fields for each section was calculated.

\section{Statistical analysis}

All data are presented as the percentage, mean and standard deviation $(\mathrm{x} \pm \mathrm{sd})$, or median and $95 \%$ confidence interval $(95 \% \mathrm{CI})$. Statistical analyses were performed using SPSS 17.0 for Windows. The two-tailed Student's t-test was used to analyze significant differences between groups. $\mathrm{P}<0.05$ indicated statistical significance.

\section{Abbreviations}

IHC: immunohistochemistry; GC: gastric cancer; NS: normal saline; EMT: epithelial-mesenchymal transition; ASCL2: Achaete-scute-like 2; MMP2: matrix metalloproteinase-2; MMP9: matrix metalloproteinase-9; CRC: colorectal cancer; ECM: Excetral Cellular Matrix; TUNEL: Terminal deoxynucleoitidyl Transferase Mediated Nick End Labeling.

\section{Author contributions}

Teng Chen and Qingshong Zuo contributed to experimental design, interpreted the data, and supervised the project. Jie Wang, Han Cai, Yue Xia performed experiments, manuscript writing and analyzed data. Peihao Yin, Shiying Wang, Likai Xing, Chao Chen, Yiming Jiang, Yong Zhang, Jie Xu, Ronghua Zhao performed the experiments.

\section{ACKNOWLEDGMENTS}

We thank all the authors that provided help for the study. We appreciate Doctor Aimee Talarski for English editing.

\section{CONFLICTS OF INTEREST}

The authors declare no conflicts of interest.

\section{FUNDING}

This work was supported by the Health System Independent Innovation Science Foundation of Shanghai Putuo District (2013PTKW002), the Foundation of Medical Discipline Priority of Shanghai Putuo District (2016PTZK01), the Foundation of the major clinical project Integrative Medicine of Shanghai (ZY3-LCPT-2-1001), and the Foundation of Doctoral Construction Program of Shanghai (B201403).

\section{REFERENCES}

1. Siegel RL, Miller KD, Jemal A. Cancer statistics, 2017. CA Cancer J Clin. 2017; 67: 7-30.

2. Chen W, Zheng R, Baade PD, Zhang S, Zeng H, Bray F, Jemal A, Yu XQ, He J. Cancer statistics in China, 2015. CA Cancer J Clin. 2016; 66: 115-132.

3. Qi X, Liu Y, Wang W, Cai D, Li W, Hui J, Liu C, Zhao Y, Li G. Management of advanced gastric cancer: an overview of major findings from meta-analysis. Oncotarget. 2016; 7: 78180-78205. https://doi.org/10.18632/oncotarget.12102.

4. Qiu DZ, Zhang ZJ, Wu WZ, Yang YK. Bufalin, a component in Chansu, inhibits proliferation and invasion 
of hepatocellular carcinoma cells. BMC Complement Altern Med. 2013; 13: 185.

5. Zhang J, Sha J, Zhou Y, Han K, Wang Y, Su Y, Yin X, Hu H, Yao Y. Bufalin inhibits proliferation and induces apoptosis in osteosarcoma cells by downregulating microRNA-221. Evid Based Complement Alternat Med. 2016; 2016: 7319464.

6. Liu X, Xiao XY, Shou QY, Yan JF, Chen L, Fu HY, Wang JC. Bufalin inhibits pancreatic cancer by inducing cell cycle arrest via the c-Myc/NF-kappaB pathway. J Ethnopharmacol. 2016; 193: 538-545. https://doi. org/10.1016/j.jep.2016.09.047.

7. Liu M, Feng LX, Sun P, Liu W, Wu WY, Jiang BH, Yang M, Hu LH, Guo DA, Liu X. A novel bufalin derivative exhibited stronger apoptosis-inducing effect than bufalin in A549 lung cancer cells and lower acute toxicity in mice. PLoS One. 2016; 11: e0159789.

8. Shen S, Zhang Y, Wang Z, Liu R, Gong X. Bufalin induces the interplay between apoptosis and autophagy in glioma cells through endoplasmic reticulum stress. Int J Biol Sci. 2014; 10: 212-224.

9. Wang H, Zhang C, Xu L, Zang K, Ning Z, Jiang F, Chi $\mathrm{H}$, Zhu X, Meng Z. Bufalin suppresses hepatocellular carcinoma invasion and metastasis by targeting HIF-1alpha via the PI3K/AKT/mTOR pathway. Oncotarget. 2016; 7: 20193-20208. https://doi.org/10.18632/oncotarget.7935.

10. Gai JQ, Sheng X, Qin JM, Sun K, Zhao W, Ni L. The effect and mechanism of bufalin on regulating hepatocellular carcinoma cell invasion and metastasis via Wnt/beta-catenin signaling pathway. Int J Oncol. 2016; 48: 338-348.

11. Yuan ZT, Shi XJ, Yuan YX, Qiu YY, Zou Y, Liu C, Yu H, $\mathrm{He} \mathrm{X}, \mathrm{Xu} \mathrm{K}$, Yin PH. Bufalin reverses ABCB1-mediated drug resistance in colorectal cancer. Oncotarget. 2017; 8: 48012-48026. https://doi.org/10.18632/oncotarget.18225.

12. Zhao H, Zhao D, Jin H, Yang X, Zhuang L, Liu T. Bufalin reverses intrinsic and acquired drug resistance to cisplatin through the AKT signaling pathway in gastric cancer cells. Mol Med Rep. 2016; 14: 1817-1822.

13. Zhao H, Zhao D, Tan G, Liu Y, Zhuang L, Liu T. Bufalin promotes apoptosis of gastric cancer by down-regulation of miR-298 targeting bax. Int J Clin Exp Med. 2015; 8: 3420-3428.

14. Wang CY, Shahi P, Huang JT, Phan NN, Sun Z, Lin YC, Lai MD, Werb Z. Systematic analysis of the achaete-scute complex-like gene signature in clinical cancer patients. Mol Clin Oncol. 2017; 6: 7-18.

15. Johnson JE, Birren SJ, Anderson DJ. Two rat homologues of Drosophila achaete-scute specifically expressed in neuronal precursors. Nature. 1990; 346: 858-861.

16. Schuijers J, Junker Jan P, Mokry M, Hatzis P, Koo BK, Sasselli V, van der Flier Laurens G, Cuppen E, van Oudenaarden A, Clevers H. Ascl2 acts as an R-spondin/ Wnt-responsive switch to control stemness in intestinal crypts. Cell Stem Cell. 2015; 16: 158-170.
17. Ziskin JL, Dunlap D, Yaylaoglu M, Fodor IK, Forrest WF, Patel R, Ge N, Hutchins GG, Pine JK, Quirke P, Koeppen $\mathrm{H}$, Jubb AM. In situ validation of an intestinal stem cell signature in colorectal cancer. Gut. 2013; 62: 1012-1023.

18. Giakountis A, Moulos P, Zarkou V, Oikonomou C, Harokopos V, Hatzigeorgiou AG, Reczko M, Hatzis P. A positive regulatory loop between a Wnt-regulated noncoding RNA and ASCL2 controls intestinal stem cell fate. Cell Rep. 2016; 15: 2588-2596.

19. Tian Y, Pan Q, Shang Y, Zhu R, Ye J, Liu Y, Zhong X, Li S, He Y, Chen L, Zhao J, Chen W, Peng Z, et al. MicroRNA-200 (miR-200) cluster regulation by achaete scute-like 2 (Ascl2): impact on the epithelial-mesenchymal transition in colon cancer cells. J Biol Chem. 2014; 289: 36101-36115.

20. Liu MH, Cui YH, Guo QN, Zhou Y. Elevated ASCL2 expression is associated with metastasis of osteosarcoma and predicts poor prognosis of the patients. Am J Cancer Res. 2016; 6: 1431-1440.

21. Hu XG, Chen L, Wang QL, Zhao XL, Tan J, Cui YH, Liu $\mathrm{XD}$, Zhang X, Bian XW. Elevated expression of ASCL2 is an independent prognostic indicator in lung squamous cell carcinoma. J Clin Pathol. 2016; 69: 313-318.

22. Kwon OH, Park JL, Baek SJ, Noh SM, Song KS, Kim SY, Kim YS. Aberrant upregulation of ASCL2 by promoter demethylation promotes the growth and resistance to 5-fluorouracil of gastric cancer cells. Cancer Sci. 2013; 104: 391-397.

23. Li C, Hashimi SM, Cao S, Mellick AS, Duan W, Good D, Wei MQ. The mechanisms of chansu in inducing efficient apoptosis in colon cancer cells. Evid Based Complement Alternat Med. 2013; 2013: 849054.

24. Zou Z, Luo X, Nie P, Wu B, Zhang T, Wei Y, Wang W, Geng G, Jiang J, Mi Y. Inhibition of SRC-3 enhances sensitivity of human cancer cells to histone deacetylase inhibitors. Biochem Biophys Res Commun. 2016; 478: 227-233.

25. Wang J, Chen C, Wang SY, Zhang Y, Yin PH, Gao ZX, Xu J, Feng DX, Zuo QS, Zhao RH, Chen T. Bufalin inhibits HCT116 colon cancer cells and its orthotopic xenograft tumor in mice model through genes related to apoptotic and PTEN/AKT pathways. Gastroenterol Res Pract. 2015; 2015: 457193.

26. Sun P, Feng LX, Zhang DM, Liu M, Liu W, Mi T, Wu WY, Jiang BH, Yang M, Hu LH, Guo DA, Liu X. Bufalin derivative BF211 inhibits proteasome activity in human lung cancer cells in vitro by inhibiting betal subunit expression and disrupting proteasome assembly. Acta Pharmacol Sin. 2016; 37: 908-918.

27. Wang H, Ning Z, Li Y, Zhu X, Meng Z. Bufalin suppresses cancer stem-like cells in gemcitabine-resistant pancreatic cancer cells via Hedgehog signaling. Mol Med Rep. 2016; 14: 1907-1914.

28. Kang KH, Han MH, Jeong JW, Park C, Lee SH, Lee HW, Hong SH, Choi YH, Hong SH. Bufalin sensitizes human 
bladder carcinoma cells to TRAIL-mediated apoptosis. Oncol Lett. 2017; 14: 853-859.

29. Zhou ZH, Rao J, Yang J, Wu F, Tan J, Xu SL, Ding Y, Zhan $\mathrm{N}, \mathrm{Hu}$ XG, Cui YH, Zhang X, Dong W, Liu XD, et al. SEMA3F prevents metastasis of colorectal cancer by PI3KAKT-dependent down-regulation of the ASCL2-CXCR4 axis. J Pathol. 2015; 236: 467-478.

30. Coghlin C, Murray GI. Current and emerging concepts in tumour metastasis. J Pathol. 2010; 222: 1-15.

31. Huang LL, Wang Z, Cao CJ, Ke ZF, Wang F, Wang R, Luo CQ, Lu X, Wang LT. AEG-1 associates with metastasis in papillary thyroid cancer through upregulation of MMP2/9. Int J Oncol. 2017; 51: 812-822.

32. Cheng TC, Din ZH, Su JH, Wu YJ, Liu CI. Sinulariolide suppresses cell migration and invasion by inhibiting matrix metalloproteinase-2/-9 and Urokinase through the PI3K/ AKT/mTOR signaling pathway in human bladder cancer cells. Mar Drugs. 2017; 15: 1-11.

33. Kalluri R, Weinberg RA. The basics of epithelialmesenchymal transition. J Clin Invest. 2009; 119: 1420-1428.

34. Lee MY, Chou CY, Tang MJ, Shen MR. Epithelialmesenchymal transition in cervical cancer: correlation with tumor progression, epidermal growth factor receptor overexpression, and snail up-regulation. Clin Cancer Res. 2008; 14: 4743-4750.
35. Garg M. Epithelial, mesenchymal and hybrid epithelial/ mesenchymal phenotypes and their clinical relevance in cancer metastasis. Expert Rev Mol Med. 2017; 19: e3.

36. Dave JM, Bayless KJ. Vimentin as an integral regulator of cell adhesion and endothelial sprouting. Microcirculation. 2014; 21: 333-344.

37. Serman L, Martic TN, Serman A, Vranic S. Epigenetic alterations of the Wnt signaling pathway in cancer: a mini review. Bosn J Basic Med Sci. 2014; 14: 191-194.

38. Ma Y, Zhu B, Liu X, Yu H, Yong L, Liu X, Shao J, Liu Z. Inhibition of oleandrin on the proliferation show and invasion of osteosarcoma cells in vitro by suppressing Wnt/ beta-catenin signaling pathway. J Exp Clin Cancer Res. 2015; 34: 115.

39. van der Flier LG, van Gijn ME, Hatzis $P$, Kujala $P$, Haegebarth A, Stange DE, Begthel H, van den Born M, Guryev V, Oving I, van Es JH, Barker N, Peters PJ, et al. Transcription factor achaete scute-like 2 controls intestinal stem cell fate. Cell. 2009; 136: 903-912.

40. Jeong YJ, Choi Y, Shin JM, Cho HJ, Kang JH, Park KK, Choe JY, Bae YS, Han SM, Kim CH, Chang HW, Chang YC. Melittin suppresses EGF-induced cell motility and invasion by inhibiting PI3K/Akt/mTOR signaling pathway in breast cancer cells. Food Chem Toxicol. 2014; 68: 218-225. 